\title{
DORVILLEIDOS (POLYCHAETA, DORVILLEIDAE) RECOLECTADOS DURANTE EL PROYECTO "FAUNA IBÉRICA"Y CATÁLOGO DE LAS ESPECIES ÍBERO-BALEARES
}

\author{
J. Núñez*, Y. Maggio* \& R. Riera**\#
}

\begin{abstract}
RESUMEN
J. Núñez, Y. Maggio \& R. Riera. 2013. Dorvilleidos (Polychaeta, Dorvilleidae) recolectados durante el proyecto "Fauna Ibérica" y catálogo de las especies íbero-baleares. Grael/sia, 69(2): 275-282.

Se elabora un listado de las especies de dorvilleidos (Eunicida, Dorvilleidae) a partir de muestras recogidas durante las campañas oceanográficas "Fauna Ibérica II, III y IV" que agrupa un total de 6 géneros y 9 especies. A partir del material estudiado, se cita por primera vez para el litoral de la península lbérica la especie comensal de crustáceos: Iphitime paguri Fage \& Legendre, 1934. También se incluye un catálogo actualizado de los dorvilleidos conocidos para el ámbito íbero-balear, compuesto por 7 géneros y 22 especies. Por último, se presenta un apéndice con datos abióticos para cada estación: localidad, coordenadas, profundidad y tipo de sustrato.
\end{abstract}

Palabras clave: Dorvilleidae; Polychaeta; Península Ibérica; catálogo de especies.

\section{ABSTRACT}

J. Núñez, Y. Maggio \& R. Riera. 2013. Dorvilleids (Polychaeta, Dorvilleidae) collected during "Fauna lbérica" project and a check-list of species recorded from the Iberian Peninsula. Graellsia, 69(2): 275-282 (in Spanish).

A check-list of Dorvilleids (Eunicida, Dorvilleidae) species collected during the oceanographic cruises "Fauna lbérica II, III and IV" is presented. A total of 6 genera and 9 species were identified, including the first record from the littoral of Iberian Peninsula of Iphitime paguri Fage \& Legendre, a species commensal of crustaceans. An updated catalogue of recorded dorvilleids from the Iberian Peninsula and Balearic islands is presented. This catalogue comprises 7 genera and 22 species. An appendix with data (locality, coordinates and seabed substrate) of each sampling station is provided.

Key words: Dorvilleidae; Polychaeta; Iberian Peninsula; check-list.

* Astrofísico Francisco Sánchez s/n, Departamento de Biología Animal (Zoología), Facultad de Biología, Universidad de La Laguna, 38206 La Laguna, Tenerife, Islas Canarias, España, e-mail: janunez@ull.es

** Centro de Investigaciones Medioambientales del Atlántico (CIMA SL), Arzobispo Elías Yanes, 44, 38206 La Laguna, Tenerife, Islas Canarias, España

\# Dirección postal actual: Department of Biodiversity, Qatar Environment and Energy Research Institute (QEERI), 5825 Doha, Qatar 


\section{Introducción}

Los poliquetos pertenecientes a la familia Dorvilleidae Chamberlin, 1919 se incluyen en el clado Eunicida (Dales, 1962), son anélidos caracterizados por la posesión de una faringe musculosa ventral, armada con un par de mandíbulas ventrales y filas de piezas maxilares en posición dorsal aunque, excepcionalmente, algunas especies pueden carecer de aparato mandibular; tanto las piezas mandibulares como maxilares presentan diferentes grados de esclerotización según su estado de desarrollo y la especie de que se trate. Otra característica que define a este grupo es la presencia de un peristomio bianillado, carente de apéndices y sedas.

El clado Eunicida, siguiendo a Rouse \& Pleijel (2001, 2007), se compone de las siguientes familias: Dorvilleidae Chamberlin, 1919, Eunicidae Berthold, 1827, Hartmaniellidae Imajima, 1977, Histriobdellidae Vaillant, 1890, Lumbrineridae Schmarda, 1861, Oenonidae Kinberg, 1865 y Onuphidae Kinberg, 1865. Fauchald (1970) define una nueva familia, Iphitimidae Fauchald, 1970 creada para un grupo de especies comensales de crustáceos decápodos que se encuentran en sus cámaras branquiales. Los aspectos morfológicos de estos anélidos los emparentaba estrechamente con los dorvilleidos, diferenciándose de ellos por la presencia de branquias dorsales bien desarrolladas. Sin embargo, estudios taxonómicos posteriores corroboran la presencia de branquias en algunos dorvilleidos eliminando tal distinción entre ambas (Rullier, 1974; Armstrong \& Jumars, 1978), siendo Gaston \& Benner (1981) los primeros que proponen que se incluyan las especies de Iphitimidae en Dorvilleidae.

Los primeros estudios de revisión taxonómica de la familia Dorvilleidae fueron los realizados por Pettibone (1961), autora que contribuye de forma significativa a un importante cambio en la sistemática de esta familia, así como los posteriores estudios de Orensanz (1973), Jumars (1974) y Oug (1978). Son de destacar asimismo los trabajos de Westheide \& Nordheim (1985) sobre dorvilleidos intersticiales en Europa, Australia y Nueva Zelanda, de Wolf (1984) en el golfo de Méjico, de Hilbig \& Blake (1991) en el talud Atlántico de EEUU y de Hilbig \& Scott (1995) en la costa de California. En la región Atlántico-Mediterránea y, en concreto, en el ámbito íbero-balear, la revisión más completa se debe a Campoy (1982) quien recopila 5 géneros y 8 especies. Actualmente, a nivel general la familia contiene alrededor de 40 géneros y unas 212 especies, siendo el $42 \%$ de los géneros monoespecíficos (Read \& Fauchald, 2013). En el litoral íbero-balear se han registrado, hasta el momento 7 géneros y 22 especies.

\section{Material y métodos}

El material estudiado proviene de las campañas oceanográficas Fauna II, III y IV, realizadas dentro del Proyecto Fauna Ibérica. Para más detalles sobre estas campañas, consultar Núñez et al. (2011).

En el presente trabajo se recopilan las especies identificadas, la estación de procedencia y el número de ejemplares colectados. Una de ellas, destacada con asterisco $(*)$ ha resultado ser el primer registro para el ámbito íbero-balear; también se incluye un listado de todas las especies citadas para el mencionado ámbito. Todo el material se encuentra depositado en el Museo Nacional de Ciencias Naturales de Madrid (MNCN).

\section{Resultados}

A continuación se relacionan en orden alfabético los géneros y especies que han sido determinados en el material estudiado. Para cada especie se incluye el nombre de la campaña, el código de la estación de muestreo y, entre paréntesis, el número de ejemplares colectados. Otros datos específicos de cada estación quedan reflejados en el Apéndice final, donde se relacionan, junto a su código, el nombre de la localidad, coordenadas iniciales y finales del muestreo, profundidad y tipo de sustrato.

\section{Familia Dorvilleidae Chamberlin, 1919}

Género Dorvillea Parfitt, 1866

Dorvillea erucaeformis (Malmgren, 1865)

REFERENCIAS EN EL ÁMBITO ÍBERO-BALEAR. Tena, 1996; Torres-Gavilá, 2008.

MATERIAL EXAMINADO. Fauna III: 186A (1), 230B18 (1). Fauna IV: $305 \mathrm{~A}(2)$.

DisCUSIÓN. Se trata de una especie que se ha considerado sinónima de Dorvillea rubrovittata, siendo redescrita de nuevo por Josefson (1975). Las características fundamentales que se aceptan para diferenciar ambas especies se basan en la forma y el tamaño de las antenas y 
palpos. En D. erucaeformis las antenas son simples (inarticuladas) y los palpos están más desarrollados que en $D$. rubrovittata. Las sedas simples del haz supra-acicular son bífidas en $D$. erucaeformis, mientras que en $D$. rubrovittata están irregularmente festoneadas. El registro de Sardá (1984; 1986) para el estrecho de Gibraltar como D. rubrovittata (non Grube, 1855), a la vista de la iconografía presentada coincide con la especie $D$. erucaeformis.

\section{Dorvillea rubrovittata (Grube, 1855)}

REFERENCIAS EN EL ÁMBITO ÍBERO-BALEAR. Rioja, 1918; 1920; Bellan, 1959; 1960; Ibáñez, 1973; Camp, 1976; Campoy, 1982; Baratech, 1985; Alós, 1988; Parapar, 1991; López, 1995; Méndez \& Cardell, 1996; Tena, 1996; Redondo \& San Martín, 1997.

Material eXAminado. Fauna II: 87DH (1), 113A (1), 138A (2). Fauna III: 179B2 (5), 194A (5), 210B11 (1), 213A (2), 222A (1), 229B2 (1), 229B7 (1), 231B1 (1), 236B3 (1), 236B14 (2), 238 (17), 238A (2), 239A (1), 250A (4), 262B2 (1), 273B1 (3). Fauna IV: 274B2 (3), 274B4 (1), 277B12 (1), 277B26 (1), 278B1 (3), 282B2 (1), 285B22 (1), 295B1 (1), 304A (1), 305A (14), 307A (4), 308B7 (1), 313A (1), 315B2 (5), 316A (1), 317A (1), 324A (1), 328B2 (1).

Género Iphitime Marenzeller, 1902

Iphitime cuenoti Fauvel, 1914

REFERENCIAS EN EL ÁMBITO ÍBERO-BALEAR. Abelló, 1985; Abelló et al., 1988.

MATERIAL EXAMINADO. Fauna II: 105A (1).

*Iphitime paguri Fage \& Legendre, 1934

MATERIAL EXAMINADO. Fauna II: 91A (6), 102A (1), 105A (4), 106A(1), 110A (1), 113A(1), 127A (1), 148A (29), 151A(12), 164A (2), 168A (14), 169A (2), 170A (1).

Discusión. Se trata de una especie conocida a lo largo de las costas Atlánticas de Francia e islas Británicas (Comely \& Ansell, 1989; Høisæter \& Samuelsen, 2006), los nuevos registros del material estudiado amplían su distribución por todo el litoral Cantábrico. Es una especie comensal que habita en las cámaras branquiales de crustáceos paguros Eupagurus bernhardus y E. prideauxi (Fage \& Legendre, 1934; Comely \& Ansell, 1989).

Género Ophryotrocha Claparède \& Mecznikow, 1869

Ophryotrocha cantabrica Núñez, Riera \& Maggio (en prensa)

REFERENCIAS EN EL ÁMBITO ÍBERO-BALEAR. Núñez et al., (en prensa)
Material eXAMinado. Fauna II: 114A (45), 150A (7).

DisCUSIÓN. Especie que sólo se conoce para dos localidades del Cantábrico: Gijón y Zumaya. Habita en fondos arenosos y arenoso fangosos entre 70-100 m de profundidad. Se caracteriza por sus antenas y palpos bien desarrollados, carecer de ojos, parápodos con un largo cirro dorsal, lóbulo acicular subtriangular y lóbulo setal inferior bien desarrollado, con una seda inferior; mandíbulas bífidas con los mangos alargados y maxilas tipo-P (Núñez et al., en prensa).

Género Pettiboneia Orensanz, 1973

Pettiboneia urciensis Campoy \& San Martín, 1980

Referencias en el Ámbito íbero-balear. Campoy \& San Martín, 1980; San Martín et al., 1981; Alós, 1988.

MATERIAL EXAMINADO. Fauna III: 183B1 (1), 211B (1).

Género Protodorvillea Pettibone, 1961

Protodorvillea kefersteini (McIntosh, 1869)

ReFERENCIAS EN El ÁmBito ÍBERO-BALEAR. Desbruyères et al., 1972; Campoy, 1982; Aguirrezabalaga, 1984; Besteiro-Rodríguez, 1986; Alós, 1988; Parapar, 1991; Méndez \& Cardell, 1996; Soler et al., 1997; Moreira-Da Rocha, 1999; Martínez \& Adarraga, 2001.

MATERIAL EXAMINADO. Fauna III: 211B (2).

Género Schistomeringos Jumars, 1974

Schistomeringos neglecta (Fauvel, 1923)

REFERENCIAS EN EL ÁMBITO ÍBERO-BALEAR. Desbruyères et al., 1972; Torres-Gavilá et al., 1990; Méndez \& Cardell, 1996; Tena, 1996; Torres-Gavilá, 2008.

MATERIAL EXAMINADO . Fauna III: 177B3 (1).

Schistomeringos rudolphii (delle Chiaje, 1828)

REFERENCIAS EN El ÁmBITO íBero-BALEAR. Rioja, 1920; Desbruyères et al., 1972; Camp, 1976; San Martín et al., 1981; Campoy, 1982; Aguirrezabalaga, 1984; Alós, 1988; Parapar, 1991; Soler et al., 1997; Méndez \& Cardell, 1996; Tena, 1996; Martínez \& Adarraga, 2001; Torres-Gavilá, 2008.

MAterial eXAminAdo . Fauna II: 158A (1). Fauna III: 203B1 (1).

Graellsia, 69(2), Diciembre 2013, pp. 275-282 - ISSN: 0367-5041 doi:10.3989/graellsia.2013.v69.095 


\section{Listado de géneros y especies citados en la Península Ibérica y Baleares}

Se incluyen a continuación los 7 géneros y 22 especies de dorvilleidos registrados para el ámbito íberobalear, destacando el género Ophryotrocha como el más diverso con 9 especies registradas. Las referencias bibliográficas para la elaboración del presente listado son: Campoy (1982), Aguirrezabalaga (1984), Ariño (1987), Abelló et al. (1988), Martín et al. (1991), Parapar (1991); Parapar et al., (1992); Villora-Moreno (1993), Méndez \& Cardell (1996), Martínez \& Adarraga (2001), Serrano-López (2002), Aguirrezabalaga \& Ceberio (2003); Torres-Gavilá (2008), Wiklund et al. (2009) y Paxton \& Åkesson $(2010 ; 2011)$.

Familia DORVILLEIDAE Chamberlin, 1919

Género Dorvillea Parfitt, 1866 Dorvillea erucaeformis (Malmgren, 1865)

Dorvillea rubrovittata (Grube, 1855)

Género Diurodrilus Remane, 1925

Diurodrilus benazzii Gerlach, 1952

Género Iphitime Marenzeller, 1902

Iphitime cuenoti Fauvel, 1914

Iphitime paguri Fage y Legendre, 1934

Género Ophryotrocha Claparède \& Mecznikow, 1869

Ophryotrocha alborana Paxton \& Åkesson, 2011

Ophryotrocha cantabrica Núñez, Riera \& Maggio, en prensa

Ophryotrocha geryonicola (Esmark, 1874) [O. mediterranea Martín, Abelló \& Cartes, 1991 syn.] Ver Discusión.

Ophryotrocha hartmanni Huth, 1933

Ophryotrocha labronica Bacci \& La Greca, 1961

Ophryotrocha macrovifera Paxton \& Åkesson, 2010

Ophryotrocha puerilis Claparède \& Mecznikow, 1869

Ophryotrocha robusta Paxton \& Åkesson, 2010

Ophryotrocha rubra Paxton \& Åkesson, 2010

Género Pettiboneia Orensanz, 1973

Pettiboneia sanmartini Aguirrezabalaga \& Ceberio, 2003

Pettiboneia urciensis Campoy \& San Martín, 1980

Género Protodorvillea Pettibone, 1961

Protodorvillea kefersteini (McIntosh, 1869)

Género Schistomeringos Jumars, 1974

Schistomeringos albomaculata (Åkesson \& Rice, 1992)

Schistomeringos anoculata (Hartman, 1965)

Schistomeringos caeca (Webster \& Benedict, 1884)

Schistomeringos neglecta (Fauvel, 1923)

Schistomeringos rudolphii (delle Chiaje, 1828)

\section{Discusión}

Se propone la sinonimia de Ophryotrocha mediterranea Martín, Abelló \& Cartes, 1991 con O. geryonicola (Esmark, 1874), ya que las diferencias morfológicas fundamentales entre ambas especies, propuestas por Martín et al. (1991), basadas en el número de piezas maxilares y la forma de las sedas, creemos no se ajustan a la realidad. Si se comparan las redescripciones de $O$. geryonicola aportadas por Wesenberg-Lund (1938) y Gaston y Benner (1981), en este caso como Eteonopsis geryonicola, con la descripción original de $O$. mediterranea de Martín et al. (1991), se comprueba que en $O$. geryonicola existe una gran variación intraespecífica tanto en el número de piezas maxilares como en su morfología según el estado de desarrollo de los ejemplares, observándose que algunos llegan a tener hasta 7 pares de piezas maxilares e incluso más (WesenbergLund, 1938), número éste de piezas maxilares descritas en la población de $O$. mediterranea estudiada por Martín et al. (1991). Por otra parte, la morfología y el número de sedas por parápodo también es similar en ambas especies, estando la ligera denticulación descrita por Martín et al. (1991) presente tanto en las sedas simples como compuestas, aunque sólo es posible apreciarla a grandes aumentos, razón por la cual en descripciones anteriores no se hace referencia a su presencia. O. geryonicola, ya había sido con anterioridad citada en aguas del mar Mediterráneo y, asociada, a las cámaras branquiales del crustáceo decápodo Geryon longipes (Desportes et al., 1977; Mori \& Belloni, 1985).

\section{Agradecimientos}

Este trabajo es una contribución dentro del Proyecto "Fauna Ibérica X, subproyecto CGL 2010-22267-C07-03" concedido por la Dirección General de Investigación. Agradecemos también a todas las personas del Museo Nacional de Ciencias Naturales de Madrid (MNCN) y, en particular, al personal que ha participado en las diversas campañas oceanográficas de Fauna II, III y IV, que nos han proporcionado todas las facilidades para la revisión del material y los datos de recolección de las muestras.

\section{Referencias}

Abelló, P., 1985. Iphitime cuenoti (Polychaeta: Iphitimidae), commensale des crabes en Méditerranée. Rapport Commission International Mer Méditerranée, 29(5): 355-356. 
Abelló, P., Sardá, R. \& Masalles, D., 1988. Infestation of some Mediterranean brachyuran crabs by the polychaete Iphitime cuenoti. Cahiers de Biologie Marine, 29: 149-162.

Aguirrezabalaga, F., 1984. Contribución al estudio de los Anélidos Poliquetos de la costa de Guipúzcoa. Munibe, San Sebastián, 36: 119-130.

Aguirrezabalaga, F. \& Ceberio, A., 2003. Dorvilleidae (Polychaeta) from the Capbreton Canyon (Bay of Biscay, NE Atlantic) with the description of Pettiboneia sanmartini sp. nov. Cahiers de Biologie Marine, 44: 41-48.

Alós, M. C., 1988. Anélidos Poliquetos del Cabo de Creus (Alt Empordá). Tesis Doctoral. Universidad de Barcelona. Barcelona. 838 pp.

Ariño, A., 1987. Bibliografía Ibérica de Poliquetos. Base de datos y catálogo de especies. Publicaciones de Biología de la Universidad de Navarra, Serie Zoológica, 16: 1-169.

Armstrong, J. W. \& Jumars, P. A., 1978. Branchiate Dorvilleidae (Polychaeta) from the North Pacific. Bulletin of the Southern California Academy of Sciences, 77(3): 133-138.

Baratech, L., 1985. Contribución al conocimiento de los Anélidos Poliquetos de las costas andaluzas. Tesis de Licenciatura, Universidad Autónoma de Madrid, Madrid. 306 pp.

Bellan, G., 1959. Campagnes de la "Calypso": Mer d'Alboran. 2. Annélides Polychètes. Annales de l'Institut Océanographique de Monaco, 37: 315-342.

Bellan, G., 1960. Annélides Polychètes. Résultats Scientifiques de la Campagne du N.R.P. "Faial» dans les eaux côtières du Portugal (1957), 2: 1-31.

Besteiro-Rodríguez, M. C., 1986. Fauna mesopsámmica de las arenas de Amphioxus de la ría de Ferrol, Galicia. Tesis Doctoral, Universidad de Santiago, Santiago de Compostela. 260 pp.

Camp, J., 1976. Comunidades bentónicas de sustrato duro del litoral NE español. IV. Poliquetos. Investigaciones Pesqueras, 40(2): 533-550.

Campoy, A., 1982. Fauna de España. Fauna de anélidos poliquetos de la Península Ibérica. 1 y 2. Publicaciones de la Universidad de Navarra. Serie Zoológica. 7: 1781.

Campoy, A. \& San Martín, G., 1980. Pettiboneia urciensis sp. n.: un nouveau Dorvilleidae (Polychètes: Errantes) de la Méditerranée. Cahiers de Biologie Marine, 21: 201-207.

Comely, C. A. \& Ansell, A. D., 1989. The occurrence of the eunicid polychaetes Iphitime cuenoti Fauvel and I. paguri Fage \& Legendre in crabs from the Scottish west coast. Ophelia, 31: 59-76.

Dales, R. P., 1962. The polychaete stomodeum and the interrelationships of the families of the Polychaeta. Proceedings of the Zoological Society of London, 139: 289-328.
Desbruyères, D., Guille, A. \& Ramos, J. M., 1972. Bionomie benthique du plateau continental de la côte catalane espagnole. Vie et Milieu, 23(2B): 335-366.

Desportes, I., Laubier, L. \& Théodoridés, J., 1977. Présence d'Ophryotrocha geryonicola (Esmark) (Polychète: Dorvilleidae) en Méditerranée occidentale. Vie et Milieu, Sér. A, 27(1): 131-133.

Fage, L. \& Legendre, R., 1934. Les annélides polychètes du genre Iphitime. A propos d'une espèce nouvelle commensale des pagures, Iphitime paguri n. sp. Bulletin de la Société zoologique de France, 58: 299305.

Fauchald, K., 1970. Polychaetous annelids of the families Eunicidae, Lumbrineridae, Iphitimidae, Arabellidae, Lysaretidae and Dorvilleidae from western Mexico. Allan Hancock Monographs in Marine Biology, 5: 1335.

Gaston, G. R. \& Benner, D. A., 1981. On Dorvilleidae and Iphitimidae (Annelida: Polychaeta) with a redescription of Eteonopsis geryonicola and a new host record. Proceedings of the Biological Society of Washington, 94: 76-87.

Hilbig, B. \& Blake, J. A., 1991. Dorvilleidae (Annelida: Polychaeta) from U. S. Atlantic slope and rise. Description of two new genera and 14 new species, with a generic revision of Ophryotrocha. Zoologica Scripta, 20(2): 147-183.

Hilbig, B \& Scott, P. H. (Eds.), 1995. Taxonomic Atlas of the Benthic Fauna of the Santa Maria Basin and the Western Santa Barbara Channel. Vol. 5. The Annelida Part 2, Polychaeta: Phyllodocida (Syllidae and Scale-bearing families), Amphinomida, and Eunicida. Santa Barbara Museum of Natural History Publications. 378 pp.

Høisæter, T., \& Samuelsen, T. J., 2006. Taxonomic and biological notes on a species of Iphitime (Polychaeta, Eunicida) associated with Pagurus prideaux from western Norway. Marine Biology Research, 2: 333354.

Ibáñez, M., 1973. Catálogo de los Anélidos Poliquetos citados en las costas españolas. Cuadernos de Ciencias Biológicas, 2(2): 121-140.

Josefson, A., 1975. Ophryotrocha longidentata sp. n. and Dorvillea erucaeformis (Malmgren) (Polychaeta, Dorvilleidae) fron the West Coast of Scandinavia. Zoologica Scripta, 4: 49-54.

Jumars, P. A., 1974. A generic revision of the Dorvilleidae (Polychaeta), with six new species from the deep North Pacific. Zoological Journal of the Linnean Society, 54(2): 101-135.

López, E., 1995. Anélidos Poliquetos de sustratos duros de las Islas Chafarinas. Tesis Doctoral. Universidad Autónoma de Madrid. Madrid. 672 pp.

Martín, D., Abelló, P. \& Cartes, J., 1991. A new species of Ophryotrocha (Polychaeta: Dorvilleidae) commensal in Geryon longipes (Crustacea: Brachyura) 
from the Western Mediterranean Sea. Journal of Natural History, 25: 279-292.

Martínez, J. \& Adarraga, I., 2001. Distribución batimétrica de comunidades macrobentónicas de sustrato blando en la plataforma continental de Guipúzcoa (golfo de Vizcaya). Boletín del Instituto Español de Oceanografia, 17(1-2): 33-48.

Méndez, N. \& Cardell, M.J., 1996. Littoral annelid polychaetes inhabiting soft bottoms of the Barcelonès (Catalonia, NE Spain). Miscelània Zoològica, 19(1): 119-147.

Moreira-Da Rocha, J., 1999. Anélidos poliquetos de sustratos blandos infralitorales de la Ensenada de Baiona (Galicia). Memoria de Licenciatura. Universidad de Vigo. Vigo. 510 pp.

Mori, M. \& Belloni, S., 1985. Distribution, abundance and infestation of Ophryotrocha geryonicola (Annelida: Dorvilleidae) in Geryon longipes (Crustacea: Decapoda: Geryonidae) of Ligurian bathyal bottoms. Oebalia, 11: 277-287.

Núñez, J., Barnich, R., Santos, L. \& Maggio, Y., 2011. Poliquetos escamosos (Annelida, Polychaeta) colectados en las campañas "Fauna II, III, IV" (Proyecto "Fauna Ibérica") y catálogo de las especies conocidas para el ámbito íbero-balear. Graellsia, 67(2): 187-197.

Núñez, J., Riera, R. \& Maggio, Y., en prensa. A new Ophryotrocha species (Polychaeta: Dorvilleidae) from circalittoral seabeds of the Cantabrian Sea (north-east Atlantic Ocean). Journal of the Marine Biological Association of the United of Kingdom, doi:10.1017/S0025315413001082

Orensanz, J. M., 1973. Los Anélidos Poliquetos de la provincia biogeográfica Argentina. III. Dorvilleidae. Physis Sección A, Buenos Aires, 32(85): 325-342.

Oug, E., 1978. New and lesser known Dorvilleidae (Annelida, Polychaeta) from Scandinavian and Northeast American waters. Sarsia, 63: 285-303.

Parapar, J., 1991. Anélidos Poliquetos bentónicos de la Ría de Ferrol (Galicia). Tesis Doctoral. Universidad de Santiago de Compostela. Santiago de Compostela. $1104 \mathrm{pp}$.

Parapar, J., Besteiro, C. \& Urgorri, V., 1992. Nuevas aportaciones al conocimiento de los anélidos poliquetos en el litoral gallego (N. O. Península Ibérica). Nova Acta Científica Compostelana (Bioloxía), 3: 109-123.

Paxton, H. \& Åkesson, B., 2010. The Ophryotrocha labronica group (Annelida: Dorvilleidae) with the description of seven new species. Zootaxa, 2713: 1-24.

Paxton, H. \& Åkesson, B., 2011. The Ophryotrocha diadema group (Annelida: Dorvilleidae), with the description of two new species. Zootaxa, 3092: 43-59.

Pettibone, M. H, 1961. New species of polychaete worms from the Atlantic Ocean, with a revision of the Dorvilleidae. Proceedings of the Biological Society of Washington, 74: 167-186.

Read, G. \& Fauchald, K., 2013. Dorvilleidae. In: Read, G. \& Fauchald, K. (Ed.) (2013) World Polychaeta database. Accessed through: World Register of Marine Species at http://www.marinespecies.org/ aphia.php? $\mathrm{p}=$ taxdetails\&id=971 on 2013-11-11

Redondo M. S. \& San Martín, G., 1997. Anélidos poliquetos de la costa comprendida entre el cabo de San Antonio y el puerto de Valencia (Mediterráneo occidental). Publicaciones Especiales del Instituto Español de Oceanografia, 23: 225-233.

Rioja, E., 1918. Datos para el conocimiento de la fauna de Anélidos Poliquetos del Cantábrico ( $2^{\mathrm{a}}$ parte). Trabajos del Museo de Ciencias Naturales, Serie Zoológica, 37: 1-99.

Rioja, E., 1920. Una campaña biológica en el Golfo de Valencia. Anales del Instituto de Genética Técnica, Valencia, 20: 1-36.

Rouse, G. W. \& Pleijel, F., 2001. Polychaetes. Oxford University Press. London. 354 pp.

Rouse, G. W. \& Pleijel, F., 2007. Annelida. En: Zhang, Z.-Q. y Shear, W.A. (Eds.). Linnaeus Tercentenary: Progress in Invertebrate Taxonomy. Zootaxa, 1668: 245-264.

Rullier, F., 1974. Quelques annelids polychètes de Cuba recueillies dans des éponges. Travaux du Muséum D' Histoire Naturelle "Grigore Antipa", 14: 9-77.

San Martín, G., Viéitez, J. M., \& Campoy, A., 1981. Contribución al estudio de la fauna de Anélidos Poliquetos de las costas españolas: poliquetos errantes recolectados en la bahía de Palma de Mallorca. Boletín del Instituto Español de Oceanografia, 6: 63-87.

Sardá, R., 1984. Contribución al conocimiento de los Anélidos Poliquetos litorales ibéricos. Estudio sobre la fauna de Anélidos Poliquetos de las zonas mediolitoral e infralitoral, en la Región del Estrecho de Gibraltar. Tesis Doctoral. Universidad de Barcelona. Barcelona. 901 pp.

Sardá, R. 1986. Fauna de Anélidos Poliquetos de la región del Estrecho de Gibraltar. III-Eunicida, Orbiniida, Spionida, Magelonida, Chaetopterida, Ctenodrilina, Flabelligerida, Opheliida, Oweniida, Capitellida, Terebellida, Sabellida y Nerillida. Miscel-lánia Zoològica, 10: 71-85.

Serrano-López, A., 2002. Ecología de las poblaciones de poliquetos del entorno de la isla de Mouro (Santander, mar Cantábrico). Tesis Doctoral. Universidad Autónoma de Madrid. Madrid. 439 pp.

Soler, A., Ballesteros, M. \& Turón, X., 1997. Poliquetos del Estany des Peix (Formentera, Baleares). Aproximación al estudio faunístico y ecológico. Historia Animalium, 3: 9-23.

Tena, J., 1996. Faunistica y ecología de los anélidos poliquetos de los fondos de sustrato duro del archipiéla- 
go de las Chafarinas (S. W. mar de Alborán). Tesis Doctoral. Universitat de València. Valencia. 549 pp.

Torres-Gavilá, F. J., 2008. Estudio faunístico, ecológico $y$ ambiental de la fauna de anélidos poliquetos de sustratos sueltos de las islas Chafarinas (Mar de Alborán, S.W. Mediterráneo). Tesis Doctoral. Universitat de València. Valencia. 695 pp.

Torres-Gavilá, F. J., Capaccioni-Azzati, R. \& GarcíaCarrascosa, A. M., 1990. Características sedimentarias y fauna de poliquetos de la desembocadura del río Segura (Mediterráneo occidental). Actas del VI Simposio Ibérico del Bentos Marino, Bilbilis, Palma de Mallorca: 81-88.

Villora-Moreno, S., 1993. Heterogeneidad del ambiente intersticial y biodiversidad de la meiofauna: el meiobentos de las islas Chafarinas. Tesis Doctoral. Universitat de València. Valencia. 325 pp.

Wesenberg-Lund, E., 1938. Ophryotrocha geryonicola (Bidenkap) (= Eteonopsis geryonicola Bidenkap) refound and redescribed. Kungliga Vetenskaps och Vitterhets-Samhälles Handlingar, ser. B, 6(8): 1-14.
Westheide, W. \& Nordheim, H. von, 1985. Interstitial Dorvilleidae (Annelida, Polychaeta) from Europe, Australia and New Zealand. Zoologica Scripta, 14(3): 183-199.

Wiklund, H., Glober, A. G \& Dahlgren, T.G., 2009. Three new species of Ophryotrocha (Annelida: Dorvilleidae) from a whale-fall in the North-East Atlantic. Zootaxa, 2228: 43-56.

Wolf, P. S., 1984. Family Dorvilleidae Chamberlin, 1919. En: Taxonomic guide to the Polychaetes of the Northern Gulf of Mexico. Uebelacker, J. M. \& Johnson, P. G. (Eds.). Barry A. Vittor \& Associates. Mobile, 44: 1-44.

Apéndice.- Estaciones de las campañas oceanográficas Fauna II, III y IV del proyecto "Fauna Ibérica" en las que se colectaron ejemplares de la Familia Dorvilleidae.

Appendix. - Sampling stations of the oceanographic cruises "Fauna Ibérica II, III and IV" where specimens of the Family Dorvilleidae were collected.

FAUNA II

\begin{tabular}{|c|c|c|c|c|c|}
\hline ESTACIÓN & LOCALIDAD & $\begin{array}{l}\text { COORDENADAS } \\
\text { INICIALES }\end{array}$ & $\begin{array}{l}\text { COORDENADAS } \\
\text { FINALES }\end{array}$ & PROF. (m) & SUSTRATO \\
\hline 87DH & S. cabo Finisterre & $42^{\circ} 49.77^{\prime} \mathrm{N}, 09^{\circ} 13.91^{\prime} \mathrm{W}$ & $42^{\circ} 49.811^{\prime} \mathrm{N}, 09^{\circ} 13.81^{\prime} \mathrm{W}$ & $54-67$ & Rocoso con braquiópodos \\
\hline $102 \mathrm{~A}$ & NO. cabo Prior & $43^{\circ} 54.48^{\prime} \mathrm{N}, 08^{\circ} 37.99^{\prime} \mathrm{W}$ & $43^{\circ} 55.68^{\prime} \mathrm{N}, 08^{\circ} 39.07^{\prime} \mathrm{W}$ & $408-435$ & Arenoso fangoso con Munida sp. \\
\hline $105 \mathrm{~A}$ & N. ría de Foz & $43^{\circ} 58.75^{\prime} \mathrm{N}, 07^{\circ} 13.12^{\prime} \mathrm{W}$ & $43^{\circ} 58.18^{\prime} \mathrm{N}, 07^{\circ} 10.48^{\prime} \mathrm{W}$ & $174-180$ & $\begin{array}{l}\text { Asociado a pagúridos y } \\
\text { portúnidos }\end{array}$ \\
\hline $106 \mathrm{~A}$ & N. ría de Foz & $43^{\circ} 55.8^{\prime} \mathrm{N}, 07^{\circ} 09.01^{\prime} \mathrm{W}$ & $43^{\circ} 53.91^{\prime} \mathrm{N}, 07^{\circ} 07.74^{\prime} \mathrm{W}$ & $158-167$ & $\begin{array}{l}\text { Asociado a pagúridos y } \\
\text { portúnidos }\end{array}$ \\
\hline $110 \mathrm{~A}$ & N. Ribadeo & & & & Asociado a pagúridos \\
\hline $113 \mathrm{~A}$ & N. Gijón & $43^{\circ} 43.57^{\prime} \mathrm{N}, 05^{\circ} 55.67^{\prime} \mathrm{W}$ & $43^{\circ} 43.85^{\prime} \mathrm{N}, 05^{\circ} 56.76^{\prime} \mathrm{W}$ & $116-120$ & $\begin{array}{l}\text { Fondo coraligeno (Dendrophyllia } \\
\text { ramea) }\end{array}$ \\
\hline $114 \mathrm{~A}$ & N. Gijón & $43^{\circ} 37.71^{\prime} \mathrm{N}, 05^{\circ} 37.59^{\prime} \mathrm{W}$ & $43^{\circ} 38.36^{\prime} \mathrm{N}, 05^{\circ} 39.78^{\prime} \mathrm{W}$ & $72-74$ & Arenoso fangoso con esponjas \\
\hline $127 \mathrm{~A}$ & N. cabo Mayor & $43^{\circ} 35.83^{\prime} \mathrm{N}, 03^{\circ} 44.32^{\prime} \mathrm{W}$ & $43^{\circ} 35.71^{\prime} \mathrm{N}, 03^{\circ} 44.45^{\prime} \mathrm{W}$ & 130 & Adansia palliata y pagúridos \\
\hline $138 \mathrm{~A}$ & NO. cabo de Ajo & & & & \\
\hline $148 \mathrm{~A}$ & N. San Sebastián & $43^{\circ} 29.56^{\prime} \mathrm{N}, 02^{\circ} 01.58^{\prime} \mathrm{W}$ & $43^{\circ} 29.88^{\prime} \mathrm{N}, 02^{\circ} 00.20^{\prime} \mathrm{W}$ & $135-143$ & Fangoso \\
\hline $151 \mathrm{~A}$ & E. cabo Machichaco & $43^{\circ} 25.29^{\prime} \mathrm{N}, 02^{\circ} 31.05^{\prime} \mathrm{W}$ & $43^{\circ} 25.09^{\prime} \mathrm{N}, 02^{\circ} 29.83^{\prime} \mathrm{W}$ & $82-86$ & Asociado a pagúridos con actinias \\
\hline $158 \mathrm{~A}$ & N. cabo Oyambre & $43^{\circ} 29.43^{\prime} \mathrm{N}, 04^{\circ} 20.89^{\prime} \mathrm{W}$ & $43^{\circ} 29.41^{\prime} \mathrm{N}, 04^{\circ} 20.68^{\prime} \mathrm{W}$ & $136-137$ & $\begin{array}{l}\text { Roca y piedras con esponjas y } \\
\text { ofiuras }\end{array}$ \\
\hline $164 \mathrm{~A}$ & NO. cabo Lastres & $43^{\circ} 40.27^{\prime} \mathrm{N}, 05^{\circ} 13.36^{\prime} \mathrm{W}$ & $43^{\circ} 40.06^{\prime} \mathrm{N}, 05^{\circ} 14.35^{\prime} \mathrm{W}$ & 146 & Asociado a pagúridos \\
\hline $168 \mathrm{~A}$ & NE. cabo Prior & $43^{\circ} 45.13^{\prime} \mathrm{N}, 08^{\circ} 10.09^{\prime} \mathrm{W}$ & $43^{\circ} 46.53^{\prime} \mathrm{N}, 08^{\circ} 09.59^{\prime} \mathrm{W}$ & $116-120$ & Arenoso con fango \\
\hline $169 \mathrm{~A}$ & N. cabo Prior & $43^{\circ} 44.50^{\prime} \mathrm{N}, 08^{\circ} 17.68^{\prime} \mathrm{W}$ & $43^{\circ} 45.52^{\prime} \mathrm{N}, 08^{\circ} 18.33^{\prime} \mathrm{W}$ & $158-164$ & Asociado a pagúridos \\
\hline $170 \mathrm{~A}$ & NE. islas Sisargas & $43^{\circ} 36.81^{\prime} \mathrm{N}, 08^{\circ} 44.37^{\prime} \mathrm{W}$ & $43^{\circ} 35.77^{\prime} \mathrm{N}, 08^{\circ} 43.19^{\prime} \mathrm{W}$ & $165-174$ & Fangoso \\
\hline
\end{tabular}


FAUNA III

\begin{tabular}{|c|c|c|c|c|c|}
\hline ESTACIÓN & LOCALIDAD & $\begin{array}{l}\text { COORDENADAS } \\
\text { INICIALES }\end{array}$ & $\begin{array}{l}\text { COORDENADAS } \\
\text { FINALES }\end{array}$ & PROF. (m) & SUSTRATO \\
\hline 177B3 & N. isla Dragonera & $39^{\circ} 35.66^{\prime} \mathrm{N}, 02^{\circ} 19.58^{\prime} \mathrm{E}$ & & 30 & Detrítico \\
\hline $186 \mathrm{~A}$ & Cabo Cros & $39^{\circ} 49.66^{\prime} \mathrm{N}, 02^{\circ} 40.78^{\prime} \mathrm{E}$ & $39^{\circ} 47.64^{\prime} \mathrm{N}, 02^{\circ} 38.71^{\prime} \mathrm{E}$ & $59-61$ & Maërl con rocas pequeñas \\
\hline $194 \mathrm{~A}$ & NE. cabo de Pera & $39^{\circ} 46.09^{\prime} \mathrm{N}, 03^{\circ} 32.04^{\prime} \mathrm{E}$ & $39^{\circ} 44.17^{\prime} \mathrm{N}, 03^{\circ} 32.45^{\prime} \mathrm{E}$ & $58-59$ & Detrítico con rocas \\
\hline 203B1 & Cabo Nati & $40^{\circ} 43.10^{\prime} \mathrm{N}, 03^{\circ} 49.42^{\prime} \mathrm{E}$ & & 31 & Detrítico, Posidonia oceanica \\
\hline 210B11 & Isla Nitge & $40^{\circ} 05.60^{\prime} \mathrm{N}, 04^{\circ} 40.40^{\prime} \mathrm{E}$ & & 9 & Pared vertical \\
\hline $211 \mathrm{~B}$ & Cala Fornells & $40^{\circ} 02.83^{\prime} \mathrm{N}, 04^{\circ} 08.04^{\prime} \mathrm{E}$ & & $0-2$ & Apnea \\
\hline 211B1 & Cala Fornells & $40^{\circ} 02.83^{\prime} \mathrm{N}, 04^{\circ} 08.04^{\prime} \mathrm{E}$ & & $0-2$ & Apnea \\
\hline $213 A$ & Punta de's Murté & $40^{\circ} 04.23^{\prime} \mathrm{N}, 04^{\circ} 11.38^{\prime} \mathrm{E}$ & $40^{\circ} 05.20^{\prime} \mathrm{N}, 04^{\circ} 08.00^{\prime} \mathrm{E}$ & $55-60$ & Coralígeno \\
\hline $222 \mathrm{~A}$ & S. Punta de la Guardia & $39^{\circ} 19.30^{\prime} \mathrm{N}, 03^{\circ} 17.20^{\prime} \mathrm{E}$ & $39^{\circ} 17.82^{\prime} \mathrm{N}, 03^{\circ} 14.16^{\prime} \mathrm{E}$ & $92-97$ & Detrítico \\
\hline 229B2 & O. cabo Blanco & $39^{\circ} 22.10^{\prime} \mathrm{N}, 02^{\circ} 46.80^{\prime} \mathrm{E}$ & & 21 & $\begin{array}{l}\text { Arenisca y algas Peysonnelia } \\
\text { squamaria }\end{array}$ \\
\hline 229B7 & O. cabo Blanco & $39^{\circ} 22.10^{\prime} \mathrm{N}, 02^{\circ} 46.80^{\prime} \mathrm{E}$ & & 10 & Algas en rocoso \\
\hline $230 \mathrm{~B} 18$ & Islote del Toro & $39^{\circ} 27.74^{\prime} \mathrm{N}, 02^{\circ} 28.42^{\prime} \mathrm{E}$ & & $35-43$ & Coralígeno en roca \\
\hline 236B3 & S. isla de Tagomago & $39^{\circ} 01.80^{\prime} \mathrm{N}, 01^{\circ} 39.14^{\prime} \mathrm{E}$ & & 29 & $\begin{array}{l}\text { Mesophyllum lichenoides entre } \\
\text { P. oceanica }\end{array}$ \\
\hline 236B14 & S. isla de Tagomago & $39^{\circ} 01.80^{\prime} \mathrm{N}, 01^{\circ} 39.14^{\prime} \mathrm{E}$ & & $35-40$ & Coralígeno en roca \\
\hline 238 & & & & & Sedimento \\
\hline $238 \mathrm{~A}$ & E. cabo Martinet & $38^{\circ} 54.07 ' \mathrm{~N}, 01^{\circ} 31.14^{\prime} \mathrm{E}$ & $38^{\circ} 56.29^{\prime} \mathrm{N}, 01^{\circ} 32.77^{\prime} \mathrm{E}$ & $55-56$ & Fango \\
\hline $239 \mathrm{~A}$ & S. isla Espardel & $38^{\circ} 42.71^{\prime} \mathrm{N}, 01^{\circ} 32.28^{\prime} \mathrm{E}$ & $38^{\circ} 44.90^{\prime} \mathrm{N}, 01^{\circ} 30.51^{\prime} \mathrm{E}$ & $57-58$ & \\
\hline $250 A$ & O. cabo Berbería & $38^{\circ} 39.27^{\prime} \mathrm{N}, 01^{\circ} 20.47^{\prime} \mathrm{E}$ & $38^{\circ} 36.70^{\prime} \mathrm{N}, 01^{\circ} 20.03^{\prime} \mathrm{E}$ & $63-66$ & Pedregoso con algas \\
\hline 262B2 & E. cala Eubarca & $39^{\circ} 04.52^{\prime} \mathrm{N}, 01^{\circ} 23.13^{\prime} \mathrm{E}$ & & 10 & $\begin{array}{l}\text { Algas (Halopteris y Padina) y } \\
\text { esponjas (Ircinia muscarium) y } \\
\text { arena gruesa }\end{array}$ \\
\hline 273B1 & N. columbrete Grande & $39^{\circ} 54.02^{\prime} \mathrm{N}, 00^{\circ} 41.15^{\prime} \mathrm{E}$ & & 47 & $\begin{array}{l}\text { Coralígeno rocoso y detrítico } \\
\text { costero; Cliona viridis y maërl }\end{array}$ \\
\hline
\end{tabular}

FAUNA IV

\begin{tabular}{|c|c|c|c|c|c|}
\hline ESTACIÓN & LOCALIDAD & $\begin{array}{l}\text { COORDENADAS } \\
\text { INICIALES }\end{array}$ & $\begin{array}{l}\text { COORDENADAS } \\
\text { FINALES }\end{array}$ & PROF. (m) & SUSTRATO \\
\hline 274B2 & Islas Columbretes & $39^{\circ} 53.56^{\prime} \mathrm{N}, 00^{\circ} 41.04^{\prime} \mathrm{E}$ & $39^{\circ} 53.59^{\prime} \mathrm{N}, 00^{\circ} 41.07^{\prime} \mathrm{E}$ & 23 & Rocoso con Pentapora fascialis \\
\hline 277B26 & Islas Columbretes & $39^{\circ} 52.78^{\prime} \mathrm{N}, 00^{\circ} 40.09^{\prime} \mathrm{E}$ & $39^{\circ} 52.72^{\prime} \mathrm{N}, 00^{\circ} 40.06^{\prime} \mathrm{E}$ & 13 & Esponja indeterminada. Halimeda \\
\hline 278B1 & Islas Columbretes & $39^{\circ} 53.56^{\prime} \mathrm{N}, 00^{\circ} 40.20^{\prime} \mathrm{E}$ & $39^{\circ} 53.50^{\prime} \mathrm{N}, 00^{\circ} 40.20^{\prime} \mathrm{E}$ & 25 & Pentapora y Halimeda \\
\hline 285B22 & Islas Columbretes & $39^{\circ} 51.27^{\prime} \mathrm{N}, 00^{\circ} 40.55^{\prime} \mathrm{E}$ & $39^{\circ} 51.27^{\prime} \mathrm{N}, 00^{\circ} 40.60^{\prime} \mathrm{E}$ & 30 & Mesophyllum \\
\hline 295B1 & Columbrete Grande & $39^{\circ} 54.02 ' \mathrm{~N}, 00^{\circ} 41.08^{\prime} \mathrm{E}$ & $39^{\circ} 53.98^{\prime} \mathrm{N}, 00^{\circ} 41.11$ 'E & 43 & $\begin{array}{l}\text { Detrítico. Aglaophenia, } \\
\text { Paramuricea y coralináceas }\end{array}$ \\
\hline $304 \mathrm{~A}$ & Isla de Alborán & $35^{\circ} 55.99^{\prime} \mathrm{N}, 03^{\circ} 01.57^{\prime} \mathrm{W}$ & $35^{\circ} 55.94^{\prime} \mathrm{N}, 03^{\circ} 01.68^{\prime} \mathrm{W}$ & $29-35$ & Detrítico con Maërl \\
\hline $305 \mathrm{~A}$ & Isla de Alborán & $35^{\circ} 55.68^{\prime} \mathrm{N}, 03^{\circ} 03.25^{\prime} \mathrm{W}$ & $35^{\circ} 55.63^{\prime} \mathrm{N}, 03^{\circ} 02.67^{\prime} \mathrm{W}$ & 33-49 & Maërl \\
\hline $307 \mathrm{~A}$ & Isla de Alborán & $35^{\circ} 55.59^{\prime} \mathrm{N}, 03^{\circ} 02.34^{\prime} \mathrm{W}$ & $35^{\circ} 55.77^{\prime} \mathrm{N}, 03^{\circ} 01.54^{\prime} \mathrm{W}$ & $37-39$ & Rocoso y Maërl \\
\hline 308B7 & Isla de Alborán & $35^{\circ} 56.49^{\prime} \mathrm{N}, 03^{\circ} 01.20^{\prime} \mathrm{W}$ & $35^{\circ} 56.49^{\prime} \mathrm{N}, 03^{\circ} 01.20^{\prime} \mathrm{W}$ & $32-34$ & $\begin{array}{l}\text { Fondo con antozoos y } \\
\text { nudibranquios }\end{array}$ \\
\hline $313 \mathrm{~A}$ & Isla de Alborán & $35^{\circ} 49.91^{\prime} \mathrm{N}, 03^{\circ} 14.63^{\prime} \mathrm{W}$ & $35^{\circ} 50.36^{\prime} \mathrm{N}, 03^{\circ} 13.72^{\prime} \mathrm{W}$ & 118 & Fangoso arenoso \\
\hline 315B2 & Isla de Alborán & $35^{\circ} 58.00^{\prime} \mathrm{N}, 02^{\circ} 58.46^{\prime} \mathrm{W}$ & $35^{\circ} 58.00^{\prime} \mathrm{N}, 02^{\circ} 58.46^{\prime} \mathrm{W}$ & 36 & Rocoso \\
\hline $316 \mathrm{~A}$ & Isla de Alborán & $35^{\circ} 50.03^{\prime} \mathrm{N}, 03^{\circ} 15.23^{\prime} \mathrm{W}$ & $35^{\circ} 49.77^{\prime} \mathrm{N}, 03^{\circ} 14.45^{\prime} \mathrm{W}$ & $90-240$ & Rocoso \\
\hline $317 \mathrm{~A}$ & Isla de Alborán & $35^{\circ} 52.49^{\prime} \mathrm{N}, 03^{\circ} 08.90^{\prime} \mathrm{W}$ & $35^{\circ} 52.31^{\prime} \mathrm{N}, 03^{\circ} 07.92^{\prime} \mathrm{W}$ & $87-213$ & Escarpe rocoso \\
\hline $324 \mathrm{~A}$ & Isla de Alborán & $35^{\circ} 57.17^{\prime} \mathrm{N}, 03^{\circ} 02.00^{\prime} \mathrm{W}$ & $35^{\circ} 57.18^{\prime} \mathrm{N}, 03^{\circ} 03.00^{\prime} \mathrm{W}$ & $51-58$ & $\begin{array}{l}\text { Maërl y agregados de } \\
\text { Neopycnodonte }\end{array}$ \\
\hline 328B2 & Isla de Alborán & $35^{\circ} 57.80^{\prime} \mathrm{N}, 02^{\circ} 58.61^{\prime} \mathrm{W}$ & & 40 & Rocoso \\
\hline
\end{tabular}

